\title{
Escala de Resistência à Mudança (RAM): Construção, Evidências Psicométricas e Versão Reduzida
}

\author{
Silvana Ligia Vincenæi - Universidade Tecnológica Federal do Paraná, Medianeira, Brasil \\ Luis Augusto de Carvalho Mendes - Faculdade Maurício de Nassau - João Pessoa, Brasil \\ Valdiney Veloso Gouveia - Universidade Federal da Paraíba, João Pessoa, Brasil \\ Dalton Francisco de Andrade - Universidade Federal de Santa Catarina, Florianópolis, Brasil
}

\begin{abstract}
Resumo
O presente estudo teve o objetivo de construir uma Escala de Resistência à Mudança (RAM) que conseguisse agrupar a base teórica existente sobre o tema, reunindo evidências de sua validade fatorial e consistência interna no contexto brasileiro, assim como analisar a adequação da versão reduzida da medida. Foram executados dois estudos. O primeiro contou com a participação de 338 empregados, em sua maioria, de empresas privadas (83\%). Estes responderam à RAM e a uma qualificação demográfica. Uma análise de eixos principais (PAF) revelou a preponderância de um fator, explicando $25,56 \%$ da variância total e consistência interna (alfa de Cronbach, $\alpha$ ) de 0,94. No Estudo 2, participaram 472 empregados, a maioria de empresas privadas (55\%). Eles responderam à RAM-20 e a questões demográficas. Os resultados confirmaram a adequação psicométrica do instrumento, podendo ser empregada como uma medida unidimensional para avaliar a resistência à mudança em ambientes organizacionais. Palavras-chave: resistência, mudança, medida, questionário, validade
\end{abstract}

\section{Resistance to Change Scale (RCS): Construction, Psychometric Evidences and Reduced Version}

\begin{abstract}
The present study aimed to develop the Resistance to Change Scale (RAM), to cover the existing theoretical basis on this subject, gathering evidences of its factorial validity and internal consistency in the Brazilian context, and to analyze the adequacy of the reduced version of the measurement. Two studies were conducted. 338 employees participated in the first study, mostly from private companies (83\%). They answered RCS and demographic profile questions. A principal component analysis (PCA) revealed the predominance of one factor, explaining $25.56 \%$ of the total variance, and internal consistency (Cronbach's alpha, $\alpha$ ) of 0.94 . In the second study, there were 472 employees, the majority from private companies (55\%). They answered RCS-20 and demographic questions. The results confirmed the psychometric adequacy of the instrument, and it can be used as a unidimensional measure to assess the resistance to change in organizational environments.
\end{abstract}

Keywords: resistance, change, measure, survey, validation

\section{Escala de Resistencia a los Cambios (RAM): Construcción, Evidencias Psicométricas y Versión Reducida}

\section{Resumen}

El presente estudio tuvo como objetivo construir una Escala de Resistencia a los Cambios (RAM) que pudiese agrupar la base teórica existente sobre el tema, reuniendo evidencias de validez factorial y consistencia interna en el contexto brasileño, así como también analizar la adecuación de la versión reducida de la medida. Se realizaron dos estudios. El primero consistió en la participación de 338 empleados, en su mayoría de empresas privadas (83\%). Estos respondieron la RAM y una calificación demográfica. Un análisis de ejes principales (PAF) reveló la preponderancia de un factor, que explica 25,56\% de la varianza total y consistencia interna (alfa de Cronbach, $\alpha$ ) de 0,94. En el segundo estudio participaron 472 empleados, la mayoría de empresas privadas $(55 \%)$ que respondieron la RAM-20 y los temas demográficos, y los resultados confirmaron la adecuación psicométrica del instrumento, pudiendo ser utilizada como una medida unidimensional para evaluar la resistencia a los cambios en ambientes organizativos.

Palabras-clave: resistencia, cambios, medir, cuestionario,validez

A resistência à mudança tem sido reconhecida há muito tempo como um fator importante que pode influenciar no sucesso ou no esforço de uma mudança organizacional. É empregada frequentemente na literatura sobre a mudança organizacional como uma explicação do porquê que esforços para introduzir mudanças em larga escala em tecnologia, métodos de produção, práticas gerenciais ou sistema de compensação são de poucas expectativas ou fracassam
(Rastekenari, Monsef, \& Majnoon, 2013; Boohene \& Williams, 2012; Erwin \& Garman, 2010; Ijaz \& Vitalis, 2011; Oreg, 2006). A resistência à mudança organizacional é uma expressão de reserva, isto é, de incerteza, de dúvida, de insegurança, de restrição, que normalmente surge como uma resposta ou uma reação à mudança (Block, 1989), ou um ato de recusar a participação em uma iniciativa de mudança, que nem sempre é aparente 
(Bareil, 2012; Appelbaum, Degbe, MacDonald, \& Nguyen-Quang, 2015).

Várias pesquisas foram desenvolvidas no intuito de verificar a implantação de mudanças em organizações (Waldersee \& Griffiths, 1997; Terziovski, Sohal, \& Moss, 1997; Eisen, Mulraney, \& Sohal, 1992; Oakland $\&$ Sohal, 1987). Essas pesquisas revelaram que a resistência é uma das razões que dificultam a implementação de mudanças, resultando as tentativas em frustração e, às vezes, em fracassos (Lines, Sullivan, Smithwick, \& Mischung, 2015; Appelbaum et al., 2015; Jaramillo, Mulki, Onyemah, \& Pesquera, 2012; Ijaz \& Vitalis, 2011; Erwin \& Garman, 2010; Eaton, 2010; Kotter, 2001; Mabin, Forgeson, \& Green, 2001; Bovey \& Hede, 2001a; Maurer 1997; Spiker \& Lesser, 1995), representando não somente um custo financeiro enorme às organizações, mas desperdício de tempo e desgaste nas relações intraorganizacionais (demissões, aumento de conflitos, etc.) (Kotter, 2001; Maurer 1997).

Trabalhos têm sido desenvolvidos no sentido de propor técnicas, formas de como superar essa resistência (Zafar \& Naveed, 2014; Yılmaz \& Kiliçoğlu, 2013; Gonçalves \& Gonçalves, 2012; García-Cabrera, Álamo-Vera, \& Hernández, 2011; Hon, Bloom, \& Crant, 2011; Rafferty \& Jimmieson, 2010; Fiedler, 2010; Rosenberg, 1993; Blake, 1992; Poe \& Viator, 1990; Caruth, Middlebrook, \& Rachel, 1985; Iskatt \& Liebowitz, 1996; Kotter \& Schlesinger, 1979). Entretanto elas ainda falham em prevenir a ocorrência de resistência e, também, não têm obtido sucesso expressivo em superá-la. Poucos trabalhos têm sido publicados no sentido de medir, avaliar a resistência à mudança (Giangreco \& Peccei, 2005; Oreg, 2003; Giangreco, 2002; Judson, 1980).

Estimar possíveis atitudes ou sentimentos de resistência pode ser um passo muito útil, pois a partir dos esforços para estimar esses sentimentos, a gerência poderá estar em condições de apontar muitos problemas que poderiam surgir por imposição da mudança ou da maneira de instituí-la. O conhecimento dessa previsão é importante ao gerente no sentido de organizar seus planos para introduzir uma mudança caso queira obter sucesso. É possível, com auxílio dessa estimativa, planejar e executar contramedidas que, pelo menos, ajudarão a minimizar a resistência caso ela surja, e, quiçá, consiga transformá-la em forma de aceitação.

$O$ presente artigo objetiva construir um instrumento para avaliar a resistência a uma mudança organizacional utilizando o sistema de construção proposto por Pasquali (2009, 1999 e 1998). O instrumento obtido pode permitir aos gestores identificar, conhecer e entender as atitudes dos seus funcionários frente a uma possível mudança organizacional, considerando resistência como uma atitude que compreende os componentes afetivo, cognitivo e comportamental (Piderit, 2000). O conceito de Resistência será definido de forma mais detalhada a seguir.

\section{Resistência à Mudança}

A resistência à mudança é vista como um fenômeno natural, mas entendida como uma inimiga da mudança, pronta a aparecer durante as transformações ou inovações nas organizações, dificultando sua implementação, bem como o seu sucesso (Yue, 2008; Coghlan, 1993; Kurtz \& Duncan, 1998; Matejka \& Julian, 1993), embora alguns pesquisadores adotem uma perspectiva mais positiva, percebendo a resistência à mudança como um fenômeno natural que pode ser benéfico para uma organização (Tavakoli, 2010; Van den Heuvel \& Schalk, 2009; Mabin et al., 2001; Wadell \& Sohal, 1998).

No contexto organizacional, a resistência é normalmente testemunhada pelo gerente, como quaisquer ações percebidas do empregado na tentativa de parar, retardar ou alterar a mudança (Ford \& Ford, 2010; Bemmels \& Reshef, 1991), ou também qualquer comportamento percebido dos membros da organização que parecem relutantes em aceitar ou ajudar a implementar uma mudança organizacional (Coghlan, 1993).

Entre outros estudos que abordaram o conceito de resistência relacionados ao comportamento no ambiente organizacional, citam-se o de Brower e Abolafia (1995), Shapiro, Lewick e Devine (1995), Armenakis, Harris e Mossholder (1993). Por exemplo, Brower e Abolafia (1995) definem a resistência como uma espécie particular de ação e inação intencional que desafia, opõe às regras ou rotinas de uma organização. Já, Shapiro et al. (1995) sugerem que os empregados usam táticas enganadoras para interromper uma mudança, constituindo uma resistência à mudança. Armenakis et al. (1993) também definem a resistência em termos comportamentais, mas sugerem que outro estado a preceda: um estado cognitivo, que eles chamam de prontidão.

Watson (1971, p.745) define a resistência como "todas as forças que contribuem para a estabilidade nos sistemas da personalidade ou social". Ele salienta que a resistência à mudança tem sido, por vezes, mal interpretada como simples inércia na natureza humana, mas que, na verdade, quase todos estão ansiosos por alguma espécie de mudança de vida ou situação que gostariam 
de melhorar, por exemplo, mais dinheiro e mais liberdade para satisfazerem seus desejos. Segundo o mesmo autor, o motivo pelo qual as pessoas e as organizações não mudam deve-se às forças naturais em direção à inovação que as impedem de mudar, ou são bloqueados por forças antagônicas (Watson, 1971). Desse modo, ela é qualquer reação natural (Coghlan, 1993; Conner 1995; Motta, 1998; Zaltman \& Duncan, 1977) contra qualquer coisa que cause esse tipo de quebra nas expectativas das pessoas, de perda de equilíbrio (Conner, 1995), ou qualquer forma de perda, pois envolve frequentemente, passar do conhecido ao desconhecido (Coghlan, 1993; Myers \& Robbins, 1991; Steinburg, 1992), permitindo o avanço de alguma coisa que é familiar (Coghlan, 1993).

Uma revisão de literatura realizada por Wadell e Sohal (1998) mostrou que a resistência é vista ainda como um fenômeno complexo, de múltiplas facetas que persiste em afetar os resultados da mudança, tanto de forma negativa quanto positiva. A resistência à mudança é vista em alguns estudos como multidimensional, que inclui dimensões cognitivas, afetivas e comportamentais (Szabla, 2007; Oreg, 2003, 2006; Piderit, 2000). Para Piderit (2000), a resistência à mudança é definida como uma atitude ambivalente na resposta inicial do empregado para com a mudança. A autora empregou a visão "tripartite" de atitudes da psicologia social. A dimensão cognitiva refere-se às avaliações do indivíduo e as consequentes crenças sobre a mudança e aos seus efeitos e consiste no que ele pensa. A dimensão afetiva ou emocional envolve sentimentos e emoções de um indivíduo na resposta à mudança. Já a dimensão comportamental ou intencional refere-se a tomar alguma ação, isto é, apoiar ou opor-se, com base em comportamentos passados ou intenções futuras de agir em relação à mudança.

Oreg (2003) observou no seu estudo que as pessoas diferem umas das outras por meio da disposição interna para resistir ou aprovar mudanças, de forma que essas diferenças podem prever atitudes pessoais em relação a mudanças específicas, tanto voluntárias como impostas. Oreg (2006) conceitua a disposição para a resistência à mudança como um traço de personalidade estável, onde as pessoas que têm essa disposição são menos predispostas a incorporar as mudanças voluntariamente e, quando estas são impostas, são mais propensas a experimentar reações emocionais negativas, como ansiedade, raiva e medo.

A percepção de que a resistência pode desempenhar um papel extraordinário num esforço de mudança organizacional, seguramente se coloca em contradição a uma mentalidade que a veria como um obstáculo num processo de mudança bem-sucedido. Desse modo, manifestação de qualquer resistência pode ser um sintoma de problema inerente às situações particulares $\mathrm{e}$ serve como um sinal de aviso (Judson, 1980) de que algo não vai bem e chama a atenção aos aspectos da mudança que pode ser inapropriada, não bem pensada ou talvez simplesmente errada. De forma que os sinais de resistência em uma organização social são úteis e, se for gerenciado com cuidado, podem ser utilizadas pela organização para melhor dar assistência à mudança (Ijaz \& Vitalis, 2011; Ford \& Ford, 2010; Wadell \& Sohal, 1998; Lawrence, 1978).

A resistência à mudança se manifesta de diversas formas, umas mais aparentes ou sutis do que outras, mas todas provocam efeitos negativos para a organização (Mendes, 2001). As reações afetivas dos empregados, devidas às incertezas geradas pela mudança, podem se manifestar por meio da ansiedade, agitação e até mesmo depressão, recusa, confusão, falta de confiança, inércia e medo (Oreg, 2006; Bordia, Hunt, Paulsen, Tourish, \& Difonzo, 2004; Piderit 2000). Fiedler (2010) e Hultman (1995) observam que há duas categorias diferenciadas de resistência: ativa e passiva. A resistência ativa inclui: censurar, ridicularizar, apelar para o medo, manipular, espalhar rumores, sabotar. $\mathrm{Na}$ resistência passiva, inclui concordar verbalmente com a mudança, mas não a executa, finge ignorância, retém informações. Além dessas duas categorias de comportamentos Judson (1980), Giangreco (2002) identifica mais outros dois comportamentos: apoio (cooperação voluntária) e indiferença. Lines, Sullivan, Smithwick e Mischung, (2015) apresenta uma tabela com doze comportamentos de resistência à mudança, entre eles: fazer o mínimo possível, falta de entusiasmo; voltar às práticas tradicionais durante a implementação; saída voluntária ou involuntária do projeto ou organização.

\section{Medindo a Resistência à Mudança}

Alguns instrumentos têm sido utilizados para avaliar o grau de resistência que as pessoas apresentam no processo de mudança organizacional, Bovey e Hede (2001a, 2001b) em seus estudos utilizaram uma escala com 20 itens para medir a intenção de comportamento de resistência à mudança organizacional. Os itens são respondidos em uma escala intervalar de sete pontos, em que um (1) indicava baixa resistência e sete (7) indicava máxima resistência. O instrumento apresentou-se dividido em três fatores nomeados suporte para a mudança, resistência disfarçada e neutralidade 
passiva, com consistência interna (alfa de Cronbach) total de 0,87. As pesquisas indicaram uma associação positiva da intensão de resistência à mudança com cinco mecanismos de defesa, sendo eles, proteção, ação, isolamento de afeto, dissociação e negação (Bovey \& Hede, 2001b) e ainda com ideias irracionais (Bovey \& Hede, 2001a).

Giangreco (2002) utilizou uma escala de 13 itens para avaliar a resistência à mudança. $\mathrm{O}$ instrumento apresentou-se dividido em dois fatores, sendo o primeiro nomeado de comportamentos pró-mudança, composto por sete itens, variância explicada de 33,84\% e alfa de 0,88 , enquanto o segundo fator, comportamentos antimudanças, é composto por cinco itens, variância explicada de 19,29\% e alfa de 0,72, um item foi descartado por ter carga fatorial baixa nos dois fatores.

Um dos instrumentos mais citados na literatura foi construído por Oreg (2003). A Escala de Resistência à Mudança (Resistance to Change Scale - RTC) é composta por 17 itens e divididos em quatro fatores, sendo eles: busca por rotina $(\alpha=0,74)$, reação emocional à mudança $(\alpha=0,75)$, rigidez cognitiva $(\alpha=0,74)$ e foco no curto prazo $(\alpha=0,84)$, apresentando um alfa total de 0,87 . Além da versão original, aplicada nos Estados Unidos, a estrutura fatorial foi testada e validada em 17 países, que representaram 13 línguas em quatro continentes (Oreg et al., 2008). Esse instrumento tem sido utilizado em pesquisa como a orientação individual acerca da mudança, como determinante para escolhas e interesses profissionais (Oreg, Nevo, Metzer, Leder, \& Castro, 2009) e a percepção da justiça organizacional, que foi associada com o compromisso de mudança organizacional (Foster, 2010).

$\mathrm{Na}$ continuação de suas pesquisas sobre fatores de resistência à mudança, Oreg (2006) propôs um modelo de resistência à mudança. No modelo, as variáveis da personalidade e contexto são consideradas possíveis antecedentes da resistência. $\mathrm{O}$ autor procurou correlacionar a resistência com variáveis de resultados relacionadas ao trabalho, como a satisfação no trabalho, o comprometimento organizacional e a intenção em sair da organização (empresa). Para desenvolver esse trabalho, utilizou um instrumento de avaliação das características desses processos, o instrumento é composto por onze itens que avaliam o ambiente em que as mudanças acontecem. A escala é composta por três fatores, sendo eles: acesso à informação, com quatro itens e confiabilidade de $\alpha=0,91$, participação, com quatro itens e $\alpha=0,91$, e confiança na administração, com três itens e $\alpha=0,77$. A maior parte desses itens foi utilizada em pesquisas anteriores como Miller, Johnson e Grau (1994) e Wanberg e Banas (2000).

Para analisar as características individuais dos funcionários dentro das organizações, Oreg (2006) construiu um instrumento para avaliar as atitudes frente às mudanças. $\mathrm{O}$ modelo possui 15 itens caracterizados em componentes cognitivos (cinco itens, $\alpha=0,86$ ), afetivos (cinco itens, $\alpha=0,78$ ) e comportamentais (cinco itens, $\alpha=0,77)$. Os resultados mostraram que essas atitudes, no geral, estão associadas à satisfação com o trabalho, com o comprometimento organizacional e a intenção de sair da organização.

Schiffer (2011) propôs um instrumento para avaliar as respostas de resistência aos processos de mudança, a versão final é composto por 41 itens (alfa total de 0,98 ) organizada em quatro fatores que indicam os tipos de respostas de resistência às mudanças ocorridas no âmbito organizacional. As reações apresentaram divididas nos fatores cognitivos $(\alpha=0,92)$, afetivos $(\alpha=$ $0,97)$, comportamentais $(\alpha=0,94)$ e físicos $(\alpha=0,96)$.

No Brasil, instrumentos de pesquisa de resistência à mudança também têm sido testados e validados. Marques, Guimarães e Morais (2011) construíram um instrumento para avaliar a resistência à mudança em instituições públicas. O questionário possui 15 itens distribuídos em cinco fatores, sendo eles a decisão prévia $(\alpha=0,84)$, convívio social $(\alpha=0,80)$, resistência organizacional $(\alpha=0,85)$, experiência anterior $(\alpha=0,75)$ e aceitação e apoio individual $(\alpha=0,93)$. Além desse instrumento, Nascimento (2012) utilizou um questionário composto por 22 itens, que apresentou uma solução unifatorial como a de melhor ajuste, com um alfa de Cronbach de 0,89 e capacidade explicativa de $30,7 \%$ da variância total. Ainda, no Brasil, Neiva, Ros e $\mathrm{Paz}$ (2004) propuseram e validaram uma escala de atitudes frente à mudança organizacional. $\mathrm{O}$ instrumento continha inicialmente 50 itens, mas, na análise fatorial, permaneceram 36 itens no questionário, distribuídos em três fatores: ceticismo, $\alpha=0,95$; temores $\alpha=0,88$; e aceitação, $\alpha=0,83$.

\section{Construção da Escala}

A escala proposta para à resistência a mudança considera-a como um construto atitudinal, expressando um conjunto de pensamentos, sentimentos e formas de agir que implicam estar mais ou menos disposto a endossar ou a se adequar às mudanças em geral e que possa ser aplicado tanto em organizações públicas como privadas. Essa escala trará vantagens para as organizações e gestores nacionais, ao fornecer um instrumento 
de avaliação que considera a resistência como uma atitude, sendo capaz de levantar as suas causas, além de prever as reações e comportamentos de funcionários diante de uma proposta de mudança, constituindo um diagnóstico de resistência à mudança. Os gestores da mudança, de posse de informações obtidas com o instrumento, ao interpretar as respostas dos empregados às propostas de mudança, seriam sensibilizados nas diferentes formas nas quais a resistência pode se manifestar. Ao descobrir tais resistências, seriam capazes de apontar medidas para suavizá-las.

Para a construção da escala de resistência à mudança organizacional, foram usados os procedimentos propostos por Pasquali (1998). Os procedimentos teóricos compreenderam a revisão de literatura de resistência à mudança organizacional com a finalidade de identificar as causas (indicadores de resistência) e definir um modelo teórico. Em seguida, buscaram-se as demarcações do traço latente por meio das definições constitutivas e operacionais, a operacionalização do conceito em itens e, por fim, a análise teórica dos itens com a validação de conteúdo pelos especialistas e a análise semântica. Para isso, procedeu-se uma busca de artigos nacionais e internacionais, com os termos "resistência", "mudança" e seus correlatos em inglês, em sistemas de buscas acadêmicas como Google Acadêmico, Periódicos Capes, Sage Journals, Emerald, Springer Link, Wiley Online Library, Science Direct, EBSCO e SciELO.

Como indicadores de resistência à mudança organizacional, para definição do modelo teórico, foram escolhidos os seguintes termos, por terem sido os mais mencionados na literatura pesquisada: medo, participação, informação, resiliência psicológica, ceticismo em relação à mudança, motivação intrínseca, abertura à experiência e confiança na gerência (Zafar \& Naveed, 2014; Boohene \& Williams, 2012; Gonçalves \& Gonçalves, 2012; Saksvik \& Hetland, 2009; Van den Heuvel \& Schalk, 2009; Ashforth \& Lee, 1990; Judge Thoresen, Pucik, \& Welbourne, 1999; Kanter, 1985; Keneth, 1995; Kotter \& Schlesinger, 1979; Lawrence, 1978; Miller, Johnson, \& Grau, 1994; O’Connor, 1993; Hackman \& Oldham, 1980; Oreg, 2006; Robbins, 2002; Stanley, Meyer, \& Topolnytsky, 2005; Taylor, 1988; Tichy, 1983; Zaltman \& Duncan, 1977; Wanberg \& Banas, 2000).

$\mathrm{Na}$ conceitualização do modelo da resistência à mudança, os indicadores foram distribuídos nos componentes afetivo, cognitivo e comportamental, conforme o modelo de Oreg (2006). Além de utilizar esses indicadores selecionados, o modelo foi construído levando em consideração o conteúdo dos comportamentos, isto é, as formas de resistência diante de uma mudança: aceitação, indiferença e resistência (passiva e ativa). Após a definição do modelo conceitual, buscou-se realizar as definições constitutivas de cada indicador, como a seguir:

Medo ou receio em relação à mudança. Medo é uma reação emocional forte de alerta, desprazerosa, demonstrado pelo receio de fazer alguma coisa, geralmente por se sentir ameaçado ou tomar conhecimento de algo desagradável, pertubador, perigoso, tanto física como psicologicamente. Esse medo pode estar relacionado com medo de perder o controle das funções, perder as relações sociais do trabalho ou de não ser competente (Chaplin, 1981; Lima, 1970).

Abertura à experiência. Fator associado com uma série de comportamentos exploratórios e envolvimento com novas experiências. Pessoas que possuem uma alta abertura à experiência se mostram propensas a manifestar uma maior curiosidade, imaginação, inteligência, abertura a novas ideias, interesses culturais, educacionais, aptidão e criatividade, bem como um interesse em variadas experiências sensoriais e cognitivas, tolerância (Costa \& Widiger, 1993).

Participação na mudança. Tomar parte ou envolver-se numa atividade, maior envolvimento do empregado (embora limitada) nas decisões empresariais que os afetam diretamente (Arnold, Eysenk, \& Meili, 1982). Esse é um princípio fundamental das modernas teorias de motivação, as quais sustentam que a verdadeira motivação se dá, com intensidade e solidez, à medida que ele tenha condições de participar do planejamento, organização, liderança, controle e projetos grupais (Toledo \& Miloni, 1986).

Recompensa intrínseca. A recompensa intrínseca consiste na tendência inerente de procurar novidade e desafios, de estender as capacidades pessoais explorar, investigar e aprender. Herzberg (1966) descreveu tarefas, como motivação intrínseca quando são caracterizados por fatores denominados de "motivators", tais como reconhecimento, responsabilidade, desafio, realização, variedade, e oportunidade de avanço (Hackman \& Oldhan, 1980).

Confiança na gerência. Confiar significa ter uma relação de dependência, capacidade de inspirar nos empregados um senso de confiança, transmitir um clima de confiança para gerar uma sensação de que os empregados podem contar com seus supervisores ou gerente para fazer o que é melhor para a empresa e seus membros (Kotter, 2012; Zander, 1977). 
Resiliência psicológica. Consiste na capacidade que um indivíduo tem em tratar com problemas, superar obstáculos, ter uma alta habilidade de lidar e absorver altos níveis de mudança sem se estressar e, também, na habilidade de se ajustar a novas situações. Em Psicologia, resiliência consiste em resistir à pressão de situações adversas, como choque, estresse, etc, sem entrar em surto psicológico. É uma junção de fatores que proporcionam ao ser humano condições para enfrentar e superar problemas e adversidades em qualquer que seja a situação (Kanter, 1985; Judge et al., 1999; Wanberg \& Banas, 2000; Zaltman \& Duncan, 1977).

Informação. Consiste no modo como as informações recebidas sobre mudanças e como elas irão afetá-los e também a organização em que trabalham (Wanberg \& Banas, 2000). São instruções passadas sob a forma verbal ou escritas ou todo material utilizado para conscientizar, motivar ou agregar conhecimento que tenha relação com o trabalho, nesse caso, relativo às mudanças do trabalho (Prazeres, 1994).

Ceticismo em relação à mudança. Compreende dúvidas sobre os métodos empregados na mudança, ou numa atitude de dúvida sistemática, ou tendência a duvidar; não aceitação de pretensas certezas ou verdades absolutas sobre a eficácia da mudança, dúvida sobre as verdadeiras razões da mudança (Stanley et al. 2005).

A partir dessas definições, buscou-se criar uma escala de resistência à mudança que atendesse aos parâmetros definidos na literatura internacional e condizentes com as pesquisas realizadas no Brasil. Os procedimentos empíricos tiveram o intuito de obter respostas de juízes e de uma amostra de respondentes para auxiliar a seleção dos itens e obter estimativas dos parâmetros psicológicos da medida, como descrito nos estudos a seguir.

Estudo 1 - Evidências de Validade Fatorial e Consistência da RAM

Nesse estudo, buscou-se construir e checar as evidências da validade fatorial e consistência interna da Escala de Resistência à Mudança (RAM). Para a construção do instrumento, foram elaborados 79 itens derivados da literatura pertinente ao tema em nível internacional. Esses itens foram submetidos a cinco juízes da área de Psicologia, um deles psicometrista, e um especialista em mudança organizacional. Do total de itens, 28 tiveram porcentagem de concordância inferior a 80\% (Pasquali, 1999) e Índice de Validade de Conteúdo (IVC) abaixo de 0,8 (Alexandre \& Coluci, 2011; Polit \& Beck, 2007), indicando a eliminação deles.
Os 52 itens restantes foram submetidos à análise semântica, sendo examinados quanto à inteligibilidade (Pasquali, 2009). Para essa etapa, foram feitos três grupos de indivíduos, separados em diferentes níveis de escolaridade e idade. Foram sugeridas e realizadas alterações em nove itens. Depois dessa etapa, a RAM foi aplicada a uma amostra de 338 sujeitos para as análises exploratórias, conforme descrito abaixo.

\section{Método}

\section{Participantes}

Contou-se com uma amostra não probabilística de 338 respondentes, provenientes de empresas de Minas Gerais, em sua maioria $(83 \%)$, de empresas privadas. O tempo de serviço variou de 1 a 384 meses $(m=61$ meses, $D P=72)$. A idade dos respondentes variou de 14 e 59 anos $(m=30,3, D P=8,9)$. Dentre os respondentes, 55,6\% eram homens e solteiros (52\%), e 44,1\% mulheres e solteiras $(65,8 \%)$. A maior parte da amostra $(54,7 \%)$ apresentou o nível superior incompleto ou superior completo $(20,4 \%)$ como grau de escolaridade.

\section{Instrumentos}

A Escala de Resistência à Mudança (RAM) foi construída para medir o nível de envolvimento das pessoas com o processo de mudança organizacional. O instrumento é composto por 52 itens que avaliam a concordância ou discordância frente a transformações empresariais (por exemplo, Enfrentaria desafios proporcionados pelas mudanças no meu trabalho; Sou capaz de me adaptar às mudanças quando elas ocorrem). Os itens são avaliados por uma escala de quatro pontos, que varia entre $0=$ Discordo fortemente a $3=$ Concordo fortemente.

Além desse instrumento, foi utilizada uma caracterização sociodemográfica da amostra. Nela foram solicitadas informações que pretendiam unicamente descrever os participantes do estudo (por exemplo, idade, estado civil, sexo e tipo de empresa em que trabalha).

\section{Procedimento}

Após submissão e aprovação pelo Comitê de Ética em Pesquisa, os questionários foram aplicados em uma empresa privada de Minas Gerais, por bolsistas de Iniciação Científica (IC), que foram devidamente treinados e orientados a seguirem os procedimentos-padrão para coleta de dados por meio de questionários autoaplicáveis. Os participantes foram informados sobre os propósitos da pesquisa e que a participação 
seria voluntária, devendo responder individualmente aos questionários. Enfatizou-se que não existiam respostas certas ou erradas, bem como foi assegurado o anonimato da participação. Todos precisaram ler e assinar um Termo de Consentimento Livre e Esclarecido (TCLE). Os respondentes gastaram uma média de 20 minutos para responder o instrumento.

\section{Análise dos Dados}

A análise dos dados foi realizada com o SPSS (versão 18). Procurando verificar a estrutura fatorial da escala, os dados foram submetidos a uma análise de eixos principais (PAF). Nesse sentido, foram aplicadas os critérios de Kaiser (valor próprio igual ou superior a 1) e Cattell (distribuição gráfica dos valores próprios). Após a determinação de dimensionalidade da escala, calculou-se o alfa de Cronbach $(\alpha)$ e a correlação inter-item para o componente resultante.

\section{Resultados}

Inicialmente foi avaliado o poder de discriminação dos itens por meio do teste $t$ de Student comparando os dois grupos (escores superiores e inferiores) definidos pelo valor da mediana do valor total da soma dos itens. Os 52 itens apresentaram discriminação significativa ao nível de $t>1,96$ e $p<0,001$.

Quanto à estrutura da escala, as análises indicaram a adequação de se utilizar uma análise fatorial exploratória, tendo sido observados os seguintes valores: $K M O$ $=0,92 \mathrm{e}$ Teste de Esfericidade de Bartlett, $\chi^{2}$ (1326) $=$ 6216,$27 ; p<0,001$. Nesse sentido, realizou-se uma análise pelo método análise de eixos principais (PAF) sem fixar tipo de rotação ou número de fatores a extrair. Isso identificou 13 fatores com valores próprios (eigenvalue) superiores a 1 (critério de Kaiser). Autores como Tabachnick e Fidell (2013) consideram que o critério de Kaiser superestima o número de fatores, podendo gerar uma solução fatorial inflada.

Dessa forma, optou-se pelo critério de Cattell, que analisa a representação gráfica dos valores próprios, em que foi encontrado um fator se destacando de forma discrepante, evidenciando a forma de "cotovelo" a partir do segundo fator. O primeiro fator apresentou valor próprio de 13,29 e variância explicada de 25,56\%, a partir do segundo fator os demais valores próprios apresentados $(\leq 2,39$; variâncias explicadas $\leq 4,6 \%$ ) não apresentaram diferenças discrepantes entre si. Essas características atendem aos critérios de unidimensionalidade definidos por Reckase (1979) e Lord
(1980). Assim, decidiu-se por uma solução unidimensional, como indicada pelo Scree Plot (Figura 1). O fator apresentou valor próprio de 13,29, explicando 25,56\% da variância total e foi denominado de resistência à mudança.

Dos 52 itens, quatro $(03,04,46$ e 48$)$ foram eliminados por não atingirem carga fatorial mínima de 0,30 (Pasquali, 2009). Os demais 48 apresentaram consistência interna (alfa de Cronbach) de 0,90 e homogeneidade (correlação média item-total) de 0,25 , variando de $-0,01$ [entre os itens 18 (Mudanças não são nem boas e nem más) e 41 (Se mudanças acontecem, procuro confiar na capacidade da gerência em tomar decisões certas, especialmente àquelas que me afetam pessoalmente)] a 0,56 [entre os itens 49 (Se howver mudanças no meu trabalho, recuso a fazer trabalhos extras) e 50 (Se mudanças acontecem, não me sinto comprometido)]. A Tabela 1 detalha os resultados das análises da RAM.

\section{Discussões Parciais}

Ao realizar a análise pelo método de eixos principais (PAF), pode-se constatar a unidimensionalidade da medida, por meio do critério de Cattell (Scree Plot). A literatura indica que essa estrutura pode ser identificada quando o primeiro fator explica acima de $20 \%$ da variância total (Reckase, 1979) e quanto o primeiro valor próprio é grande comparado com o segundo, e se o segundo não é muito maior que os seguintes (Lord, 1980), como encontrado no presente estudo. Os indicadores de consistência interna (alfa de Cronbach e correlação inter-item) também apresentaram

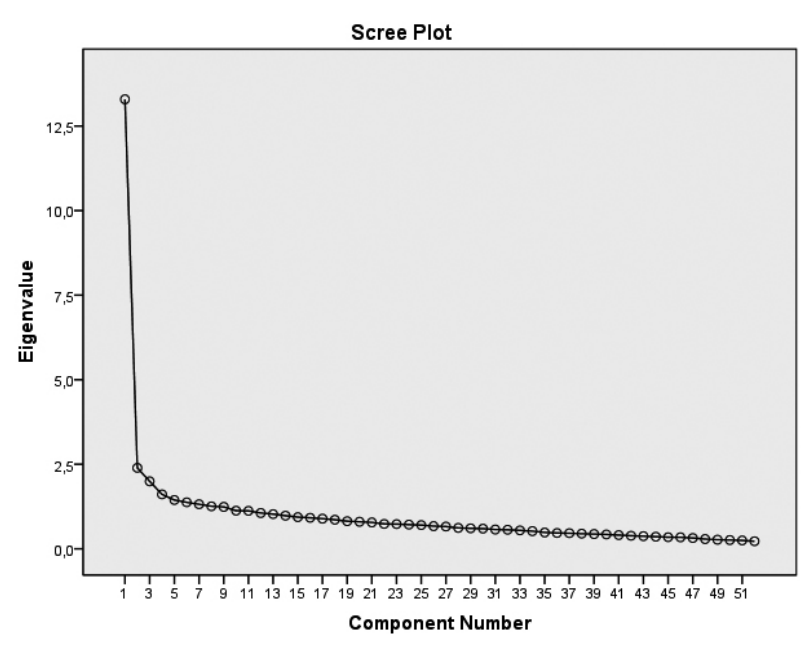

Figura 1. Distribuição gráfica dos valores próprios. 
Tabela 1

Estrutura Fatorial da Escala de Resistência a Mudança (RAM)

\begin{tabular}{lc}
\hline Itens & Fator I \\
\hline 37. Não estou interessado em realizar atividades que resultarão em mudanças. & 0,67 \\
34. Sinto que a mudança é uma ameaça. & 0,62 \\
21. Quando mudanças acontecem, procuro não me envolver nos problemas oriundos dela, procuro não & 0,62 \\
estar envolvido em problemas que não são meus. & 0,62 \\
22. Sinto que tenho entusiasmo para lidar com mudanças no meu trabalho. (r) & 0,62 \\
47. Se alguém me pressiona para mudar alguma coisa, tendo a resistir, mesmo se a mudança pode &
\end{tabular}
beneficiar-me.

38. Prefiro fazer sempre as mesmas coisas no meu trabalho, ao invés de tentar coisas diferentes. $\quad 0,60$

25. Se mudanças são implementadas no meu trabalho, gosto de participar delas. (r) $\quad 0,60$

49. Se houver mudanças no meu trabalho, recuso a fazer trabalhos extras. $\quad 0,60$

14. Apoio as ações dos meus colegas contra mudanças que ocorrem no meu trabalho. $\quad 0,60$

28. Quando ocorrem mudanças encontro motivos para me queixar da gerência. $\quad 0,58$

10. Tento evitar as responsabilidades adicionais decorrentes de mudanças em meu trabalho. $\quad 0,58$

50. Se mudanças acontecem, não me sinto comprometido. 0,58

15. Quando mudanças acontecem, procuro fazer somente o que é necessário. $\quad 0,57$

05. Quando falam em mudanças faço de conta que não é comigo. $\quad 0,57$

43. Estou disposto a colaborar para promover mudanças no meu trabalho. (r) 0,56

08. Sinto-me extremamente irritado em situações de mudança. $\quad 0,56$

26. Considero negativas as mudanças organizacionais. $\quad 0,55$

19. Se houver mudanças, atuo sem compromisso. $\quad 0,54$

16. Prefiro ficar indiferente às mudanças. $\quad 0,54$

31. Tenho receio em contribuir com as mudanças no meu trabalho. $\quad 0,54$

11. Na iminência de uma mudança no meu trabalho, procuro formas de impedir que ela venha $\quad 0,53$ acontecer.

07. Se a mudança implica em fazer algo que não gosto, realizo as tarefas lentamente. 0,53

44. Às vezes evito mudanças no meu trabalho mesmo que sejam boas para mim. 0,53

09. Experimentaria coisas novas provenientes de mudanças no meu trabalho. ( $\mathrm{r}$ ) 0,51

40. Mesmo se todos acreditarem que as mudanças são necessárias para a sobrevivência da empresa, não $\quad 0,51$ penso que isso seja verdadeiro.

06. Coopero ativamente para realizar a mudança quando ela acontece. $(r) \quad 0,50$

42. Se eu fosse informado, que haveria uma mudança em meu local de trabalho, lidaria com a situação 0,59 com estresse.

36. Sinto-me amedrontado quando mudanças acontecem devido às incertezas geradas por uma nova $\quad 0,48$ forma de trabalhar.

02. Sou capaz de me adaptar às mudanças quando elas ocorrem. (r) $\quad 0,46$

32. Gostaria de ganhar novas experiências provenientes de mudanças no meu trabalho. (r) 0,46

35. Às vezes, recuso-me a mudar o modo como faço o meu trabalho. $\quad 0,45$

01. Enfrentaria desafios proporcionados pelas mudanças no meu trabalho. (r) $\quad 0,45$

27. Acredito que tenho estabilidade emocional para lidar com mudanças no meu trabalho. ( $r) \quad 0,45$

12. Sinto que mudanças na minha rotina prejudicam meu trabalho. 0,45

45. Caso a mudança tornasse meu trabalho mais difícil, não tentaria melhorá-lo. $\quad 0,44$ 
Tabela 1

Estrutura Fatorial da Escala de Resistência a Mudança (RAM) (Continuação)

Itens Fator I

39. A mudança é positiva para seus funcionários. (r)

51. Não tenho certeza se as mudanças melhoram a organização (empresa).

24. Se soubesse que uma mudança aconteceria no meu trabalho, ficaria tenso.

23. Acredito que a proposta de mudança é fruto de estudo e análise organizacionais para a melhoria da empresa. (r)

29. Mesmo sob pressão, coopero bem com as mudanças. (r)

13. Acredito que as mudanças são um modo de adquirir mais trabalho.

33. Sinto-me indiferente e não surpreso em relação à mudança.

17. Confio nas mudanças implementadas pela gerência. (r)

20. Acredito que os riscos de implementar uma mudança em uma empresa são maiores do que seus benefícios.

30. Fico mais propenso a aceitar uma mudança quando recebo informações sobre ela. (r)

41. Se mudanças acontecem, procuro confiar na capacidade da gerência em tomar decisões certas,

52. Acredito que a gestão pode até ter motivos para implementar quaisquer mudanças, mas nem

48. Se durante o processo de planejamento das mudanças pudesse opinar ou sugerir, as apoiaria com maior determinação. $(\mathrm{r})^{*}$

46. Não me importo com mudanças, continuo fazendo meu trabalho da mesma maneira que vinha

fazendo.*

04. Sinto que minhas ideias podem ser mais valorizadas quando ocorrem mudanças. (r)*

03. Sinto que não posso aprender com as mudanças.*

Número de itens

Valor próprio

\% de Variância Explicada

Alfa de Cronbach

Notas. (r) Item invertido. *Item eliminado por apresentar carga fatorial inferior a $0,30$.

índices satisfatórios, ambos acima de 0,70 e 0,20, respectivamente, como indicado na literatura especializada (Nunnally, 1991; Pasquali, 2009).

Apesar da maioria das escalas de resistência a mudança possuírem estruturas multifatoriais, ressalta-se que uma estrutura unifatorial também foi identificada na pesquisa de Nascimento (2012) com uma amostra brasileira, em que foi encontrado um alfa de Cronbach de 0,89 e capacidade explicativa de $30,7 \%$ da variância total do instrumento.

Verificou-se também o funcionamento adequado dos itens, com discriminação significativa entre os níveis de alta e baixa pontuação na escala. Porém, o instrumento apresentou uma estrutura final com 48 itens o que pode gerar fadiga ou desgaste caso seja necessária sua aplicação no ambiente laboral ou em conjunto com outros instrumentos. Assim, propôs-se uma medida alternativa com a quantidade reduzida de itens que gere consequentemente maior facilidade de aplicação e parcimônia. Como descrito no Estudo 2, a seguir.

\section{Estudo 2. Parâmetros Psicométricos da RAM Versão} $\underline{\text { Reduzida }}$

Esse estudo objetivou reduzir a RAM e comprovar a estrutura fatorial e consistência interna de um 
modelo mais parcimonioso, com menor quantidade de itens, conforme detalhado a seguir:

\section{Método}

\section{Participantes}

Contou-se com uma amostra não probabilística de 472 respondentes, provenientes dos estados do Paraná, Mato Grosso do Sul, Santa Catarina e Rio Grande do Sul em sua maioria de empresas privadas $(55 \%)$. O tempo de serviço variou de 0 a 336 meses ( $m=43$ meses, $D P=$ 59). A idade média foi de 28,2 anos $(D P=8,5)$. Dentre os respondentes, $51 \%$ eram homens e solteiros $(51 \%)$, e $49 \%$ mulheres e solteiras $(53 \%)$. O nível de escolaridade predominante foi o superior incompleto $(54,7 \%)$, seguido pelo superior completo $(19,5 \%)$.

\section{Instrumento}

Os participantes responderam aos mesmos instrumentos Escala de Resistência à Fadiga e caracterização sociodemográfica, descritas no Estudo 1.

\section{Procedimento}

Foram aplicados os mesmos procedimentos do primeiro estudo, com a aplicação sendo realizada por bolsistas de Iniciação Científica, e os participantes informados que a participação era voluntária, assegurado o anonimato das informações prestadas.

\section{Análises Estatísticas}

As análises exploratórias foram realizadas com o PASW (versão 18). Por meio do AMOS (versão 18), foram realizadas as análises fatoriais confirmatórias, procurando testar o modelo reduzido da RAM. Considerou-se como entrada a matriz de covariância, adotando-se o método ML (Maximum Likelihood) de estimação. Para a verificação dos ajustes pelo método de equações estruturais foram levados em conta os seguintes valores: 1) o $\chi^{2}$ (Qui-quadrado), que testa a probabilidade de o modelo teórico se ajustar aos dados; quanto maior, esse valor piora o ajustamento; 2) o Goodness-of-Fit Index (GFI) e o Adjusted Goodness-of-Fit Index (AGFI), que variam de 0 a 1 , com valores na casa dos 0,90 ou superiores, indicando um ajustamento satisfatório; 3) o Comparative Fit Index (CFI), que é um índice comparativo, adicional, de ajuste ao modelo, com valores mais próximos de 1 , indicando melhor ajuste; aceitam-se valores de 0,90 ou superiores como expressando um modelo ajustado; 4) a Root-Mean-Square Error of Approximation (RMSEA), com seu intervalo de confiança de $90 \%$ (IC90\%), sugere como ideais valores de até 0,08 , aceitando-se até 0,10 . Esses índices são amplamente considerados na literatura especializada (Byrne, 2001; Tabachnick \& Fidell, 2013).

\section{Resultados}

\section{Redução da Medida}

Para realizar a redução da RAM, foram utilizados dois critérios, sendo o primeiro a capacidade discriminativa dos itens, e a segunda, a magnitude da saturação dos itens observados no Estudo 1, sendo escolhidos os 20 itens de maior saturação. Quanto à discriminação dos itens, todos se diferenciaram na direção esperada e de forma significativa $(t>1,96 ; p<0,001)$. Dessa forma, nenhum item foi descartado mediante esse critério.

De acordo com o segundo critério, foram selecionados os 20 itens que, no primeiro estudo, apresentaram as saturações mais fortes, todos com cargas superiores a $|0,55|$, como descritos na Tabela 1. A partir dessa seleção foi realizada uma análise confirmatória com a finalidade de comprovar a estrutura unifatorial encontrada no Estudo 1.

Estrutura Fatorial e Consistência Interna da RAM-20. Como as análises anteriores indicaram uma estrutura unifatorial para a RAM, decidiu-se realizar uma análise confirmatória, como pode ser visualizado na Figura 2.

$\mathrm{Na}$ estrutura unifatorial todas as saturações (os lambdas, 1), foram estatisticamente diferentes de zero $(t>1,96, p<0,001)$, apresentando os seguintes indicadores de ajuste: $\chi^{2}(170)=386,72, p<0,001, \chi^{2} / \mathrm{gl}=$ 2,28, GFI $=0,92, A G F I=0,90, C F I=0,91$ e RMSE $A$

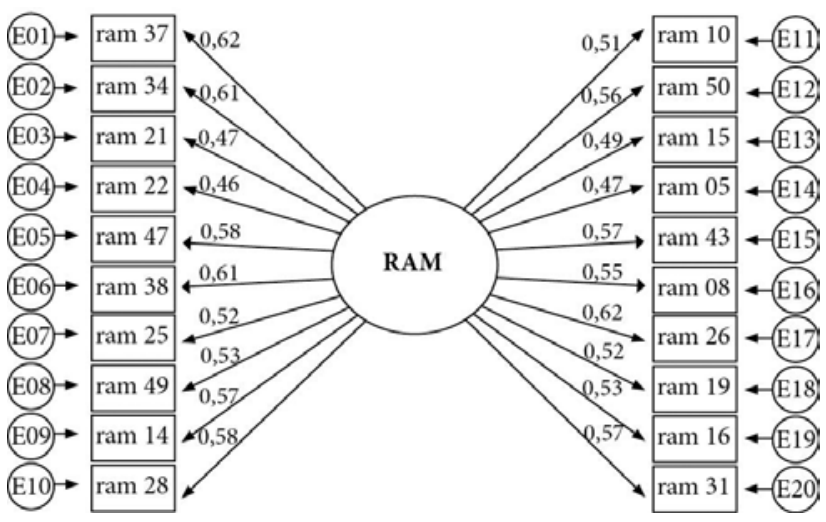

Figura 2. Estrutura unifatorial da RAM-20. 
$=0,05(0,05-0,06)$, PCLOSE $=0,31$, estando dentro das recomendações da literatura.

A estrutura apresentou consistência interna (alfa de Cronbach) de 0,89 e homogeneidade de 0,30, variando de 0,17 [entre os itens 22 (Sinto que tenho entusiasmo para lidar com mudanças no meu trabalho (r)) e 47 (Se alguém me pressiona para mudar alguma coisa, tendo a resistir, mesmo se a mudança pode beneficiar-me)] a 0,46 [entre os itens 26 (Considero negativas as mudanças organizacionais) e 34 (Sinto que a mudança é uma ameaça)].

\section{Discussão Parcial}

Diante dos resultados apresentados, podem-se considerar os objetivos do segundo estudo alcançados. Foram comprovadas a estrutura fatorial testada e a consistência interna da medida. Dessa forma, a versão com 20 itens da RAM parece aceitável, pois, embora mais parcimoniosa, reuniu parâmetros psicométricos compatíveis com aqueles sugeridos na literatura. Os coeficientes de consistência interna (alfas de Cronbach e correlação inter-item) foram superiores ao ponto de corte definido para esse parâmetro, isto é, 0,70 e 0,20 (Nunnally, 1991; Pasquali, 2009). Conjuntamente, os resultados apresentaram índices satisfatórios, indicando a escala como ajustada para a medida de resistência à mudança.

Além da verificação estatística, a RAM-20 conseguiu reunir itens que atenderam semanticamente aos principais critérios de criação da escala, com conteúdos afetivos, cognitivos e comportamentais (Oreg, 2006; Piderit, 2000) e também as formas de comportamento diante da mudança: aceitação, indiferença e resistência (passiva e ativa).

\section{Discussão Geral}

O objetivo geral desse estudo foi construir e validar uma escala de Resistência à Mudança (RAM) com amostra de empregados no contexto brasileiro, reunindo evidências de sua validade fatorial e consistência interna, além de propor um modelo reduzido do instrumento. Avaliando as análises, considera-se que este tenha sido alcançado.

Quanto à estrutura, ambos os estudos indicaram a presença de um único fator, atendendo todos os índices estabelecidos pela literatura, como a definição da unifatorialidade (Reckase, 1979; Lord, 1980) e indicadores de consistência interna, nese caso alfa de Cronbach e correlação inter-item (Nunnally, 1991; Pasquali, 2009). Da mesma forma, as analises da RAM-20 estão de acordo com o estabelecido para a confirmação de um modelo estatisticamente avaliado (Byrne, 2001; Tabachnick \& Fidell, 2013).

Pode-se citar como vantagem do modelo proposto sua facilidade de aplicação em ambientes organizacionais, visto a acessível semântica dos itens, escala de resposta clara e reduzido número de itens, principalmente no instrumento RAM-20. Questionários breves também são bem aceitos pela possibilidade de utilizá-los com outros instrumentos, diminuindo a possibilidade de fadiga ou a desistência da participação em pesquisas.

Porém, admitem-se potenciais limitações dos estudos, como o fato de ser uma amostra não probabilística, contando com representantes de poucos estados brasileiros, o que impede a generalização dos resultados para além da amostra utilizada, necessitando de estudos futuros que contem com profissionais de outras regiões do país, assim como de várias áreas de atuação. Contudo, reforça-se a natureza psicométrica dos estudos, cujas amostras foram suficientes para as análises executadas.

Finalmente, em termos de futuras direções, é necessário ampliar a aplicação da escala realizando pesquisas com outros profissionais e considerando ambientes em que as mudanças estejam acontecendo de forma evidente, o que permitiria conhecer a influência da resistência à mudança em diferentes áreas laborais, analisando se existe algum ambiente ou estratégia que se diferencie em termos de resistência, por exemplo. É importante ressaltar a importância de conhecer a relação entre o construto com outros referidos na literatura, como desperdício de tempo, conflitos ou desgastes nas relações intraorganizacionais. O que poderá facilitar na construção de ações interventivas que tenham em conta os efeitos negativos ou positivos da resistência.

\section{Referências}

Alexandre, N. M. C., \& Coluci, M. Z. O (2011). Validade de conteúdo nos processos de construção e adaptação de instrumentos de medidas. Ciência \& Saúde Coletiva, 16(7), 3061-3068.

Appelbaum, S. H., Degbe, M. C., MacDonald, O., \& Nguyen-Quang, T. S. (2015), Organizational outcomes of leadership style and resistance to change (Part One). Industrial and Commercial Training, 47(2), 73-80. doi: 10.1108/ICT-07-2013-0044 
Armenakis, A. A., Harris, S. G., \& Mossholder, K. W. (1993). Creating readiness for organizational change. Human Relations, 46, 681-703, 1993. doi: $10.1177 / 001872679304600601$

Arnold, W., Eysenk, H. J., \& Meili, R. (1982). Dicionário de Psicologia. São Paulo: Edições Loyola.

Ashforth, B. E., \& Lee, R. T. (1990). Defensive behavior in organizations: A preliminary model. Human Relations, 43, 621-648. doi: 10.1177/001872679004300702

Bareil, C. (2012). "Démystifier la résistance au changement: Questions, constats et implications sur l'expérience de changement", Changement et développement organisationnel: Faire évoluer la pratique, sous la responsabilité de Céline Bareil et Caroline Aubé, Collection: Gestion et Savoirs, HEC Montréal, 175-200.

Bemmels B., \& Reshef, Y. (1991). Manufacturing employees and technological change. Journal of Labour Research, 12(3), 231-246.

Blake, L. (1992). Reduce employees' resistance to change. Personnel Journal, 71(9), 72-75.

Block, P. (1989). "Flawless consulting". In McLennan, R. (Ed.), Managing Organizational Change. Prentice Hall: Englewood Cliffs, NJ.

Boohene, R., \& Williams A. A. (2012). Resistance to organizational change: A case study of Oti Yeboah Complex Limited. International Business and Management, 4(1), 135-145. doi: $10.3968 \% 2 \mathrm{Fj}$. ibm.1923842820120401.1040

Bordia, P., Hunt, E., Paulsen, N., Tourish, D., \& Difonzo, N. (2004). Uncertainty during organizational change: Is it all about control? European Journal of Work and Organizational Psychology, 13, 345-365. doi: 10.1080/13594320444000128

Bovey, W. H., \& Hede, A. (2001a). Resistance to organisational change: The role of defence mechanisms. Journal of Managerial Psychology, 16(7), 534-548. doi: 10.1108/EUM0000000006166

Bovey, W. H., \& Hede, A. (2001b). Resistante to organizational change: The role of cognitive and affective processes. Leadership \& organization Development Journal, 22(8), 372-382. doi: 10.1108/01437730110410099
Brower, R. S., \& Abolafia, M. Y. (1995). The structural embeddedness of resistance among public managers. Group and Organization Management, 20,149-166. doi: $10.1177 / 1059601195202005$

Byrne, B. (2001). Structural equation modeling with AMOS. Basic concepts, applications and programming. Mahwah, NJ: Lawrence Erlbaum.

Caruth, D., Middlebrook, B., \& Rachel, F. (1985). Overcoming resistance to change. S.A.M. Advanced Management Journal, 50(3), 23-27.

Chaplin, J. P. (1981). Dicionário de Psicologia. Lisboa, Publicações Dom Quixote.

Coghlan, D. (1993). A person-centred approach to dealing with resistance to change. Leadership \& Organization Development Journal, 14(4), 10-14. doi: 10.1108/01437739310039433

Conner, D. R. (1995). Gerenciando na velocidade da mudança: Como gerentes resilientes são bem sucedidos e prosperam onde outros fracassam. Tradução Andréia Alves, Rio de Janeiro: Infobook.

Costa, P. T., \& Widiger, T. A. (1993). Introduction. In P. T. Costa \& T. A. Widiger (Eds.), Personality disorders and the Five-Factor Model of Personality (pp. 1-10). Whashington, DC: American Psychological Association.

Eaton, M. (2010). "Why change programs fail". Human Resource Management International Digest. 18(2), 37-42. doi: 10.1108/09670731011028492

Eisen, H., Mulraney, B. J., \& Sohal, A. S. (1992). Impediments to the adoption of modern quality management practices. International Journal of Quality and Reliability Management, 9(5), 17-41. doi: 10.1108/EUM0000000001651

Erwin, D. G. \& Garman, A. N. (2010). Resistance to organizational change: linking research and practice. Leadership \& Organization Development Journal, 1, 3956. doi: 10.1108/01437731011010371

Fiedler, S. (2010). Managing resistance in an organizational transformation: A case study from a mobile operator company. International Journal Project Management, 28, 370-383. doi: 10.1016/j. ijproman.2010.02.004

Ford, J. D., \& Ford, L. W. (2010). Stop blaming resistance to change and start using it. Organizational Dynamics, 39(1), 24-36. doi: 10.1016/j.orgdyn.2009.10.002 
Foster, R. D. (2010). Resistance, justice, and commitment to change. Human Resource Development Quarterly, 21(1), 3-39. doi: 10.1002/hrdq.20035

García-Cabrera, A. M., Álamo-Vera, F. R., \& Hernández, F. G. B. (2011). Antecedentes de la resistência al cambio: Factores individuales y contextuales. Cuadernos de Economía y Dirección de la Empresa, 14, 231-246. doi: 10.1016/j.cede.2011.02.007

Giangreco, A., \& Peccei, R. (2005). "The nature and antecedents of middle manager resistance to change: Evidence from an Italian context". International Journal of Human Resource Management, 16(10), 18121829. doi: 10.1080/09585190500298404

Giangreco, A. (2002). Conceptualization and operationalization of resistance to change. Liuc Papers 103, Serie Economia aziendale, 11, 1-28

Gonçalves, J. M., \& Gonçalves, R. P. da S. (2012). Overcoming resistance to changes in information technology organizations. Procedia Technology, 5, 293-301. doi: 10.1016/j.protcy.2012.09.032

Hackman J., \& Oldham, G. R. (1980). Motivation through the design of work: Test of a theory. Organizational Behavior and Human Performance, 16(2), 250-279. doi: 10.1016/0030-5073(76)90016-7

Herzberg, F. (1966). Work and the nature of man. Cleveland, OH: World Publishing Company.

Hon, A. H. Y., Bloom, M., \& Crant, J. M. (2011). Overcoming resistance to change and enhancing creative performance. Journal of Management, 40(3), 919-941. doi: 10.1177/0149206311415418

Hultman, K. E. (1995). Scaling the wall of resistance. Training and Development, 49(10), 15-18.

Ijaz, S., \& Vitalis, A. (2011). Resistance to organizational change: Putting the jigsaw together. International Review of Business Research Papers, 7(3), 112-121.

Iskatt, G. J., \& Liebowitz, J. (1996). What to do when employees resist change. Supervision, 57(8), 3-5.

Jaramillo, F., Mulki, J. P., Onyemah, V., \& Pesquera, M. P. (2012). Salesperson resistance to change: an empirical investigation of antecedents and outcomes. International Journal of Bank Marketing, 30(7), 548566. doi: 10.1108/02652321211274318

Judge, T. A., Thoresen, C. J., Pucik, V., \& Welbourne, T. M. (1999). Managerial coping with organizational change: A dispositional perspective.
Journal of Applied Psychology, 84(1), 107-122. doi: 10.1037/0021-9010.84.1.107

Judson, A. S. (1980). Relações bumanas e mudanças organizacionais. São Paulo: Editora Atlas.

Kanter, R. M. (1985). Managing the human side of change. Management Review, 74, 52-56.

Keneth, H. (1995). Scaling the wall of resistance. Training \& Development, 49(10), 15-18.

Kotter, J. (2001). Oito erros fatais. In Júlio, C. \& Neto, J. (Ed.), Inovação e mudança: Autores e conceitos imprescindiveis, São Paulo: Publifolha, pp. 93-100.

Kotter, J. P. (2012). Leading change: Why transformation efforts fail. Harvard Business Review, 73(2), 59-67.

Kotter, J. P., \& Schlesinger, L. A. (1979). Choosing strategies for change. Harvard Business Review, 57(2), 106-113.

Kurtz, P., \& Duncan, A. (1998). Shared service centres: Overcoming resistance to implementation of a shared services centre. Management Accounting, 76(7), 47-48.

Lawrence, P. R. (1978). How to deal with resistance to change. Harvard Business Review, 32(3), 49-57.

Lines, B. C., Sullivan, K. S., Smithwick, J. B., \& Mischung, J. (2015). Overcoming resistanceto change in engineering and construction: Change management factors for owner organizations. International Journal of Project Management, 33, 1170-1179. doi: 10.1016/j.ijproman.2015.01.008

Lima, L. P. (1970). Dicionário de Psicologia Prática. São Paulo: Honor Editorial Ltda.

Lord, F. M. (1980). Applications of Item Response Theory to Practical Testing Problems. New York: Routledge.

Mabin, V., Forgeson, S., \& Green, L., 2001. Harnessing resistance: Using the theory of constraints to assist change management. J. Eur. Ind. Train. 25, 168-191. doi: 10.1108/EUM0000000005446

Marques, A. L., Guimarães, R. S., \& Morais, K. (2011). Validando um modelo de medida de resistência à mudança em organizações públicas. Anais do EnGPR 2011 - III Encontro de Gestão de Pessoas e Relações de Trabalho. João Pessoa, 20 a 22 de novembro de 2011. 
Matejka, K., \& Julian, R. (1993). Resistance to Change is Natural. Supervisory Mangement, 38(10), 10.

Maurer, R. (1997). Transforming Resistance. HR Focus, 74(10), 9-10.

Mendes, J. A. (2001). Resistência às tecnologias de informação, causas, efeitos e estratégias de superação: Estudo de caso da implementação do SAP R/3 (Dissertação de mestrado). Universidade do Minho, Braga.

Miller, V. D., Johnson, J. R., \& Grau, J. (1994). Antecedents to willingness to participate in a planned organizational change. Journal of $A p$ plied Communication Research, 22(1), 59-80. doi: 10.1080/00909889409365387

Motta. P. R. (1998). Transformação organizacional. A teoria e prática de inovar. Rio de Janeiro: Qualitymark.

Myers, K., \& Robbins, M. (1991). 10 rules for change. Executive Excellence, 8(5), 9-10.

Nascimento, D. A. M. (2012) Resistência à mudança organizacional: Correlatos valorativos e organizacionais (Tese de doutorado). Universidade Federal da Paraíba, João Pessoa, PB, Brasil.

Neiva, E. R., Ros, M., \& Paz, M. G. T. (2004). Validacion de una escala de actitudes ante el cambio organizacional. Revista de Psicologia Del Trabajo y das Organizaciones, 20(1), 9-30.

Nunnally, J. C. (1991). Teoría psicométrica. México, DF: Trillas.

O'connor, C. A. (1993). Resistance: The repercussions of change. Leadership \& Organization Development Journal,14(6), 30. doi: 10.1108/01437739310145615

Oakland, J., \& Sohal, A. S. (1987). Production management techniques in UK manufacturing industry: Usage and barriers. International Journal of Operations and Production Management, 7(1), 8-37. doi: 10.1108/ eb054783

Oreg, S. (2003). Resistance to change: Developing an individual difference measure. Journal of Applied Psychology, 88, 680-693. doi: 10.1037/0021-9010.88.4.680

Oreg, S. (2006). Personality, context and resistance to organizational change. European Journal of Work and Organizational Psychology, 15, 73-101. doi: 10.1080/13594320500451247
Oreg, S., et al. (2008). Dispositional resistance to change: Measurement equivalence and the link to personal values across 17 nations. Journal of Applied Psychology, 93(4), 935-944. doi: 10.1037/0021-9010.93.4.935

Oreg, S., Nevo, O., Metzer, H., Leder, N., \& Castro, D. (2009). Dispositional resistance to change and occupational interests and choices. Journal of Career Assessment, 17(3), 312-323. doi: $10.1177 / 1069072708330599$

Pasquali, L (1999). Instumentos Psicológicos: Manual prático de elaboração. Brasilia: LabPAM/IBAPP.

Pasquali, L. (1998). Princípios de elaboração de escalas psicológicas. Revista de psiquiatria clínica, 25(5), 206-213.

Pasquali, L. (2009). Psicometria: Teoria dos testes na Psicologia e na Educação. 3 ed. Petrópolis, RJ: Vozes.

Piderit, S. K. (2000). Rethinking resistance and recognizing ambivalence: A multidimensional view of attitudes toward an organizational change. Academy of Management Review, 25(4), 783-794. doi: 10.5465/ AMR.2000.3707722

Poe, C. D., \& Viator, R. E. (1990). What to do when employees resist automation. Journal of Accounting and EDP, 5(4), 9-14.

Polit, D. F., Beck, C. T., \& Owen, S. V. (2007). Is the CVI an acceptable indicator of content validity? Appraisal and recommendations. Research in nursing \& health, 30(4), 459-467. doi: 10.1002/nur.20199

Prazeres, P. M. (1994). Dicionário de termos da qualidade. São Paulo: Ed. Atlas.

Rafferty, A. E., \& Jimmieson, N. L. (2010). Team change climate: a group-level analysis of the relationships among change information and change participation, role stressors, and well-being. European Journal of Work and Organizational Psychology, 19, 551-586. doi: 10.1080/13594320903007869

Rastekenari, M. A., Monsef, S. M. S., \& Majnoon, K. (2013). Study of factors associated with employees' resistance to change and its relation with customer responsiveness and outcome performance in private Banks of rasht, Singaporean. Journal of Business Economics, And Management Studies, 2(5), 57-66.

Reckase, M. D. (1979). Unifactor latent trait models applied to multifactor tests: Results and implications. 
Journal of Educational Statistics, 4, 207-230. doi: 10.3102/10769986004003207

Robbins, S. P. (2002). Comportamento organizacional. São Paulo: Pretice Hall.

Rosenberg, D. (1993). Eliminating resistance to change. Security Management, 37(1), 20-21.

Saksvik, I. B., \& Hetland, H. (2009). Exploring dispositional resistance to change. Journal of Leadership \& Organizational Studies, 16(2), 175-183. doi: $10.1177 / 1548051809335357$

Schiffer, E. F. (2011). Resistance to change: Implications of individual differences. In Expression of Resistance to Change. [Thesis] Department of Psychology, College of Arts and Sciences. Florida: University of South Florida.

Shapiro, D. L., Lewick, R. J., \& Devine, P. (1995). When do employees choose deceptive tactics to stop unwanted organizational change? Research on Negotiation in Organizations, 5, 155-184.

Spiker, B., \& Lesser, E. (1995). Change management: We have met the enemy... Journal of Business Strategy, 16(2), 17-21. doi: 10.1108/eb039686

Stanley, D. J., Meyer, J. P., \& Topolnytsky, L. (2005). Employee cynicism and resistance to organizational change. Journal of Business and Psychology, 19(4), 429-459. doi: 10.1007/s10869-005-4518-2

Steinburg, C. (1992). Taking charge of change. Training \& Development, 46(3), 26-32.

Szabla, D. B. (2007). A multidimensional view of resistance to organizational change: Exploring cognitive, emotional, and intentional responses to planned change across perceived change leadership strategies. Human Resource Development.Quarterly, 18(4), 525-558. doi: 10.1002/hrdq.1218

Tabachnick, B. G., \& Fidell, L. S. (2013). Using Multivariate Statistics, 6 ed. Boston: Allyn and Bacon.

Tavakoli, M. (2010). A positive approach to stress, resistance, and organizational change. Procedia Social and Behavioral Sciences 5, 1794-1798. doi: 10.1016/j. sbspro.2010.07.366

Taylor, R. E. (1988). Reducing resistance to new marketing strategies. Business Forum, 13 (2), 12-15.

Terziovski, M., Sohal, A. S., \& Moss, S. (1997). A longitudunal study of quality management practices in australian organizations. Department of Management. Melbourne: Monash University.

Tichy, N. M. (1983). Managing strategic change: Technical, political, and cultural dynamics. New York: Wiley.

Toledo, F., \& Miloni, B. (1986). Dicionário de recursos bumanos. São Paulo, Ed. Atlas.

Van den Heuvel, S., \& Schalk, R. (2009). The relationship between fulfilment of the psychological contract and resistance to change during organizational transformations. Social Science Information, 48, 283-313. doi: 10.1177/0539018409102415

Wadell, D., \& Sohal, A. (1998). Resistance: A constructive tool for change management. Management Decision, 36(8), 543-548. doi: 10.1108/00251749810232628

Waldersee, R., \& Griffiths, A. (1997). The changing face of organizational change. Updaed ed. Sydney: Centre for Corporate Change, Australian Graduate School of Management, The University of New South Wales.

Wanberg, C. R., \& Banas, J. T. (2000). Predictors and outcomes of openness to changes in a reorganizing workplace. Journal of Applied Psychology, 85(1), 132-142. doi: 10.1037/0021-9010.85.1.132

Watson, G. (1971). Resistance to change. The American Behavioral Scientist, 14, 745-766.

Yılmaz, D., \& Kilıçoğlu, G. (2013). Resistance to change and ways of reducing resistance in educational organizations. European Journal of Research on Education, 1, 14-21.

Yue, W. (2008). Resistance, the echo of change. International Journal of Business and Management, 3(2), 84-89. doi: 10.5539/ijbm.v3n2p84

Zafar, F., \& Naveed, K. (2014). Organizational change and dealing with employees resistance. International Journal of Management Excellence, 2(3), 237-246. doi: 10.17722/ijme.v2i3.101.

Zaltman, G., \& Duncan, R. (1977). Strategies for planned change. New York: Wiley.

Zander, A. (1977). Resistência às modificações: Análise e prevenção. In Y. F. BALCÃO \& L. L. Cordeiro, 3. ed. O comportamento bumano na empresa. (pp. 371-80). Rio de Janeiro: Fundação Getúlio Vargas.

Recebido 16/02/2015

Reformulado 14/08/2015

Aceito 01/10/2015 
Sobre os autores:

Silvana Ligia Vincenzi é graduada em Ciências, Matemática (UNIPAR/PR) e Física (FAFICOP/PR), mestre e doutora (UFSC/SC) em Engenharia de Produção. É professora e pesquisadora da UTFPR/MD/PR, participa do Programa de Pós-Graduação em Tecnologias Computacionais para o Agronegócio e Programa de Pós-Graduação em Métodos e Gestão em Avaliação - UFSC/SC. Possui experiência na área de Probabilidade e Estatística, com ênfase em Análise de dados.

E-mail: sligie@globo.com

Luís Augusto de Carvalho Mendes é graduado em Comunicação Social, mestre e doutor em Psicologia Social (UFPB). É professor das Faculdades Estácio e Maurício de Nassau e da Universidade Federal da Paraíba, João Pessoa, $\mathrm{PB}$, atua nas áreas de pesquisa em Psicologia Social, Marketing e Comportamento do Consumidor e possui experiência profissional em empresas como os Correios e Telégrafos e Banco do Brasil.

E-mail: luisaugustomendes@gmail.com

Valdiney Veloso Gouveia é doutor em Psicologia Social pela Universidade Complutense de Madri, professor titular de Psicologia Social na Universidade Federal da Paraíba e pesquisador 1A do CNPq.

E-mail:vvgouveia@gmail.com

Dalton Francisco de Andrade é graduado em Licenciatura Matemática (USP), mestre em Estatística (USP) e doutor em Biostatistics - University of North Carolina System. É professor voluntário junto aos programas de pós-graduação PPGEP, do Departamento de Engenharia de Produção e do PPGMGA, do INE/UFSC e pesquisador associado da Fundação Vunesp. Tem experiência na área de Probabilidade e Estatística, com ênfase em Análise de Dados.

E-mail: dalton.andrade@ufsc.br

Contato com os autores:

Silvana Ligia Vincenzi

Av. Brasil, 4232, Parque Independência

Caixa Postal 271

CEP: 85884-000

Medianeira-PR, Brasil

Fone (44) 99835726 